\title{
Avaliação das respostas em frequências naturais de um violão pelo método de excitação por impulso e deconvolução de sinais
}

\author{
Evaluation of the natural frequency responses of a guitar by the method of Impulse excitation and \\ signal deconvolution \\ Evaluación de las respuestas en frecuencias naturales de una guitarra por el método de excitación \\ por impulso y desconvolución de señales
}

Recebido: 19/01/2021 | Revisado: 23/01/2021 | Aceito: 25/01/2021 | Publicado: 31/01/2021

\author{
Paulo Sérgio Teixeira \\ ORCID: https://orcid.org/0000-0002-2581-7047 \\ Universidade Federal Fluminense, Brasil \\ E-mail: paulost39@gmail.com \\ Alexandre Furtado Ferreira \\ ORCID: https://orcid.org/0000-0002-7397-7344 \\ Universidade Federal Fluminense, Brasil \\ E-mail: alexandrefurtado@id.uff.br \\ José Flávio Silveira Feiteira \\ ORCID: https://orcid.org/0000-0002-6358-9049 \\ Universidade Federal Fluminense, Brasil \\ E-mail: zeflavio@gmail.com
}

\begin{abstract}
Resumo
As partes que compõem o violão são feitas dos mais variados materiais para atender as necessidades referentes a cada componente, conforme suas funções específicas a serem desempenhadas no instrumento. Um dos importantes aspectos da pesquisa atual em acústica musical consiste na conexão das propriedades físicas mensuráveis de um instrumento musical e sua qualidade sonora ou tonal. O processamento de sinais acústicos de um instrumento de corda, por exemplo, pode correlacionar os sinais ou sons do violão com uma operação matemática de convolução ou deconvolução estabelecendo relações com suas propriedades físicas. Outra técnica utilizada é a obtenção de respostas em termos de frequência de vibração ou domínio do tempo e a técnica de excitação por impulso. No presente trabalho objetiva-se avaliar através da separação do sinal referente à resposta em frequência do violão através da deconvolução do sinal sonoro gerado ao tocar o instrumento pelo sinal das cordas gravado separadamente. Busca-se também estabelecer as relações com as respostas obtidas através da técnica de excitação por impulso. E os resultados mostram que essas duas técnicas podem representar importantes técnicas de caracterização de materiais usados em instrumentos de corda como o violão.
\end{abstract}

Palavras-chave: Violão; Deconvolução; Excitação por impulso; Frequências; Materiais.

\begin{abstract}
The parts that make up the guitar are made of the most varied materials to meet the needs related to each component, according to their specific functions to be performed on the instrument. One of the important aspects of current research in musical acoustics is the connection of the measurable physical properties of a musical instrument and its sound or tonal quality. The processing of acoustic signals from a string instrument, for example, can correlate the acoustic signals or sounds of the guitar with a mathematical operation of convolution or deconvolution by establishing relationships with its physical properties. Another technique used is to obtain responses in terms of frequency of vibration or time domain and the impulse excitation technique. The present work aims to evaluate through the separation of the signal referring to the frequency response of the guitar through the deconvolution of the sound signal generated when playing the instrument by the string signal recorded separately. It also seeks to establish the relationships with the responses obtained through the impulse excitation technique. And the results show that these two techniques can represent important techniques for characterizing materials used in string instruments such as the guitar.
\end{abstract}

Keywords: Guitar; Deconvolution; Impulse excitation; Frequencies; Materials.

\section{Resumen}

Las partes que componen la guitarra están fabricadas con los más variados materiales para cubrir las necesidades relacionadas con cada componente, de acuerdo con sus funciones específicas a realizar en el instrumento. Uno de los aspectos importantes de la investigación actual en acústica musical es la conexión de las propiedades físicas 
mensurables de un instrumento musical y su sonido o calidad tonal. El procesamiento de señales acústicas de un instrumento de cuerda, por ejemplo, puede correlacionar las señales acústicas o sonidos de la guitarra con una operación matemática de convolución o deconvolución estableciendo relaciones con sus propiedades físicas. Otra técnica utilizada es la obtención de respuestas en términos de frecuencia de vibración o dominio del tiempo y la técnica de excitación por impulsos. El presente trabajo tiene como objetivo evaluar a través de la separación de la señal referente a la respuesta en frecuencia de la guitarra mediante la deconvolución de la señal sonora generada al tocar el instrumento por la señal de cuerda grabada por separado. También busca establecer las relaciones con las respuestas obtenidas mediante la técnica de excitación por impulso. Y los resultados muestran que estas dos técnicas pueden representar técnicas importantes para caracterizar materiales utilizados en instrumentos de cuerda como la guitarra.

Palabras clave: Guitarra; Deconvolución; Excitación por impulso; Frecuencias; Materiales.

\section{Introdução}

As partes que compõem o violão são feitas dos mais variados materiais para atender as necessidades referentes a cada componente, conforme suas funções específicas a serem desempenhadas no instrumento. (Wright, 1996; Portela, 2014)

A pesquisa em acústica musical tem abrangido diversas áreas do conhecimento. São encontrados trabalhos que discorrem desde as propriedades físicas de materiais usados na construção de instrumentos de corda (Carcagno et al., 2018), até complexas análises psicoacústicas (Wright, 1996). Com isso, muitas técnicas inovadoras têm sido desenvolvidas e, aliadas ao poder de processamento dos computadores atuais, alcançando resultados surpreendentes. Elejabarrieta et al. (2000) estudou a evolução do comportamento vibracional de uma caixa acústica ou de ressonância do violão ao longo de sucessivas fases de construção por meio da técnica de análise modal. Ludwigsen (2013) comparou o som de emitido por vários violões através de características espectrais. As validações de alguns trabalhos se deram através do uso de sensores acelerômetros (Baqersad, 2014 e Avitabile, 2014) para a análise modal experimental da caixa acústica.

Um dos importantes aspectos da pesquisa atual em acústica musical consiste na conexão das propriedades físicas mensuráveis de um instrumento musical e sua qualidade sonora ou tonal. Um melhor entendimento das relações entre qualidade tonal e a resposta vibroacústica do instrumento permitirá aos fabricantes de instrumentos o ajuste das características tonais do mesmo alterando seus materiais, geometrias e padrões de construção usados.

Algumas técnicas têm sido empregadas no estudo das respostas sonoras de instrumentos de corda, entre elas, o processamento de sinais acústicos onde se pode, por exemplo, correlacionar os sinais ou sons do violão com uma operação matemática de convolução ou deconvolução estabelecendo relações com suas propriedades físicas. Outra técnica utilizada é a obtenção de respostas em termos de frequência de vibração a partir de sinais no domínio do tempo e a técnica de excitação por impulso.

Um sinal pode ser formalmente definido como uma função de uma ou mais variáveis, a qual carrega informações sobre a natureza de um fenômeno físico. Para a geração, extração de informação ou recepção de cada sinal há sempre um sistema envolvido, isto é, uma entidade capaz de manipular um ou mais sinais para realizar uma função. Por exemplo, quando alguém fala, o sistema para emissão da voz são as cordas vocais, e o ouvido é o sistema para recepção do sinal (Haykin, 2001). Assim, pode-se dizer que um sistema é capaz alterar as características de um sinal, caso desejado.

O sinal sonoro ou som resulta da vibração de um meio ou perturbação mecânica que se propaga através de ondas quando é produzido algum tipo de mecanismo que altere de forma periódica da pressão do ar em nossa volta. (Wuensche, 2009).

De acordo com Roederer (1998 apud Mendes et al., 2020) para que haja som, é necessário fazer vibrar um meio. A vibração se espalha pelo ar através das partículas que o formam, gerando áreas de maior e menor pressão, respectivamente áreas de compressão e rarefação, em movimento recíproco, uma vez que o meio é elástico. Portanto, as partículas do meio não são transmitidas junto com o som através do ambiente, que é propagada é a energia vibracional que deu origem ao som. 
Para Lazzarini (1998), o som é uma qualidade perceptiva que é o resultado da percepção de distúrbios das moléculas de um meio em certo espaço de tempo. Esses distúrbios, por sua vez, apresentam-se em forma de ondas em sua propagação pelo meio.

Segundo Lacerda (1996), o som pode ser representado por uma soma de diversas ondas individuais chamadas de componentes de Fourier e cada uma corresponde a uma determinada frequência múltipla da componente inicial.

Essas componentes formam uma série conhecida como série harmônica, onde o harmônico de ordem zero é chamado de frequência natural ou fundamental, o segundo é denominado harmônico de primeira ordem ou primeiro harmônico, o terceiro é chamado segundo harmônico e assim sucessivamente.

Para analisar os sinais acústicos de instrumentos, neste caso, o violão, é necessário considerá-lo com um sistema vibrante. Os sistemas de vibração podem vibrar em vários modos diferentes. Cada modo é relacionado a uma frequência diferente e, portanto, um modo de vibração pode ser excitado individualmente por algum tipo de perturbação relacionado a certa frequência (Santos et al., 2013).

A descrição deste movimento vibracional é bastante difícil, pois é necessário saber a amplitude (ou intensidade), a frequência de cada modo de vibração perturbado e a taxa de amortecimento no meio material em que se propaga.

$\mathrm{O}$ espectro de uma vibração indica quais frequências estão sendo excitadas e com que intensidade. Esta análise espectral, também conhecida com análise no domínio da frequência, é obtida através da análise de Fourier do sinal sonoro, especificamente pela aplicação da transformada de Fourier ao sinal temporal representativo da vibração estudada (Teixeira et al., 2014).

A convolução é um operador linear que, a partir de duas funções dadas, resulta numa terceira que mede a área subentendida pela superposição das mesmas em função do deslocamento existente entre elas (Katznelson, 1976). Neste contexto, a idéia é que a excitação da corda seja o sinal de entrada, a resposta do instrumento o segundo sinal e o sinal sonoro emitido o terceiro, que neste caso, seria o produto da convolução dos dois primeiros (Teixeira et al., 2019).

A convolução é um processo útil porque descreve com precisão alguns efeitos que ocorrem amplamente em medições científicas. Um exemplo é a influência de um filtro de frequência em um sinal elétrico ou da passagem de banda espectral de um espectrômetro na forma de um espectro óptico registrado, que causa o sinal a ser espalhado no tempo e reduzido na amplitude do pico (O’Haver, 2008).

Segundo Katznelson (1976), convolução é um operador linear que, a partir de duas funções dadas $f$ e $g$, resulta numa terceira $h(x)$ que mede a área subentendida pela superposição das mesmas em função do deslocamento existente entre elas (eq. 1). O teorema da convolução estabelece que a transformada de Fourier de uma convolução de duas funções é igual ao produto ponto a ponto das transformadas de Fourier de cada função. Em outras palavras, convolução em um domínio (no domínio do tempo) equivale à multiplicação ponto a ponto no outro domínio (no domínio da frequência).

Dadas as funções $f$ e $g$ cujo produto $h(x)=f(x) g(x)$, a convolução entre $f$ e $g$, denotada por $f_{*} g$, é definida por qualquer uma das integrais (eq. 1):

$$
h(x)=\left(f_{*} g\right)(x)=\int_{-\infty}^{+\infty} f(x-\omega) g(\omega) d \omega=\int_{-\infty}^{+\infty} f(\omega) g(x-\omega) d \omega
$$

Muito usada na geologia, a deconvolução busca a melhoria da resolução temporal do dado sísmico por meio da diminuição da contribuição do pulso enviado pela fonte. Na prática, a deconvolução retira o efeito borrado do dado sísmico e torna as reflexões na subsuperfície muito mais evidentes na imagem da seção geológica gerada (Yilmaz, 1987). 
Em matemática, deconvolução é um algoritmo baseado no processo usado para reverter os efeitos da convolução nos dados registrados. O conceito de deconvolução é amplamente utilizado em técnicas de processamento de sinais e de processamento de imagem. Estas técnicas são amplamente utilizadas em muitas aplicações de engenharia. Em geral, o objetivo da deconvolução é encontrar a solução de uma equação de convolução da forma, de acordo com O'Haver, 2008:

$$
\left(f_{*} g\right)=h
$$

Normalmente, h (eq. 2) é algum sinal gravado, e $f$ é algum sinal que queremos recuperar, mas foi convoluido com algum outro g sinal. A função g pode representar a função de transferência de um instrumento ou a força motriz que foi aplicada a um sistema físico. Se soubermos g, ou pelo menos saber a forma de g, então pode realizar a deconvolução. No entanto, se não sabemos g de antecedência, precisamos estimá-lo. Isso geralmente é feito através de métodos de estimativa estatística. Em medições físicas, a situação é geralmente próxima.

$$
\left(f_{*} g\right)+\varepsilon=h
$$

Neste caso, $\varepsilon$ é o ruído (eq. 3) que entrou nosso sinal gravado. Se assumirmos que um sinal ruidoso ou imagem é silencioso quando tentamos fazer uma estimativa estatística de $g$, a nossa estimativa será incorreta. Por sua vez, a nossa estimativa de $f$ também será incorreta. Quanto menor for a relação sinal ruído, pior será a estimativa do sinal deconvoluido, (O’Haver, 2008).

A técnica de excitação por impulso consiste em produzir um leve impacto ao espécime, neste caso, no tampo do violão, mais precisamente no cavalete, que após um curto período transitório, irá vibrar em uma ou mais de suas frequências ressonantes. A técnica de excitação por impulso é essencialmente não destrutivo e não requer nenhum formato especial para o objeto testado. E têm tido grande aceitação mundial tanto na indústria e na pesquisa (Roebben et al., 1997).

Os modos de vibração de um espécime analisado dependem de sua forma, o local do impacto e o tipo de suporte. O local do impacto é próximo a um antinodo da vibração. O impacto pode ser fornecido manualmente, com uma ferramenta simples com uma ponta metálica ou através de dispositivo automático de impacto. A vibração da amostra é registrada por um cristal piezoelétrico ou por um microfone. Um cristal piezoelétrico requer contato com o objeto analisado e para se ter uma boa medição deve se estabelecer o lugar de contato o mais próximo possível do nó de vibração (Roebben et al., 1997).

A Técnica de Excitação por Impulso usada no presente trabalho foi desenvolvida pela empresa Sonelastic de acordo com a Norma Astm E1876 (2007). As frequências são excitadas por meio de um impacto mecânico de curta duração, seguido da captação da resposta acústica por um sensor. Um tratamento matemático é feito no sinal para a obtenção do espectro de frequências (Transformada Rápida de Fourier).

No presente trabalho objetiva-se avaliar através da separação do sinal referente à resposta em frequência do violão através da deconvolução do sinal sonoro gerado ao tocar o instrumento pelo sinal das cordas gravado separadamente. Busca-se também estabelecer as relações com as respostas obtidas através da técnica de excitação por impulso.

\section{Metodologia}

\subsection{Caracterização da Resposta em Frequência pela Excitação por impulso}

O violão analisado neste trabalho é da marca Barros que segue o modelo clássico, desenvolvido pelo luthier brasileiro Antônio Maurício Barros (Figura 1 a) que tomou como base para o modelo clássico do luthier alemão Hermmam Hauser com uma configuração diferente de leque harmônico.

A análise de caso de aspecto quantitativo (Pereira et al., 2018) no presente trabalho utiliza-se da técnica de excitação por impulso através do software sonelastic (Otani et al., 2017), que consiste neste caso, em promover um leve impacto no 
cavalete do violão ao lado posição de cada corda do instrumento captando as respostas em frequências naturais através de microfone (Figura 1 b).

Figura 1 - (a) Violão pendurado no suporte; (b) Excitação por impulso na ponte nas posições da primeira a sexta corda (da esquerda para a direita, de cima para baixo).

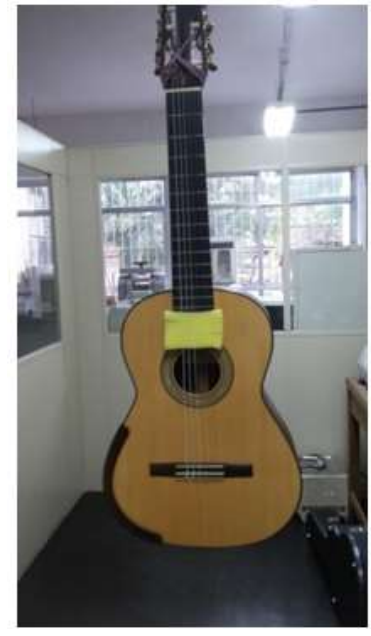

(a)
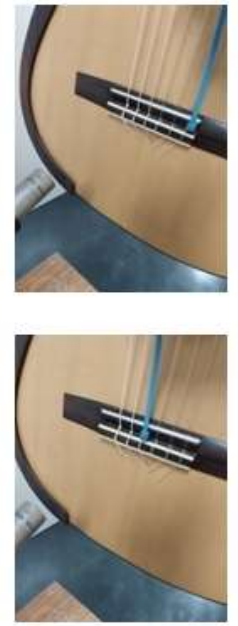

Fonte: Autores (2020).
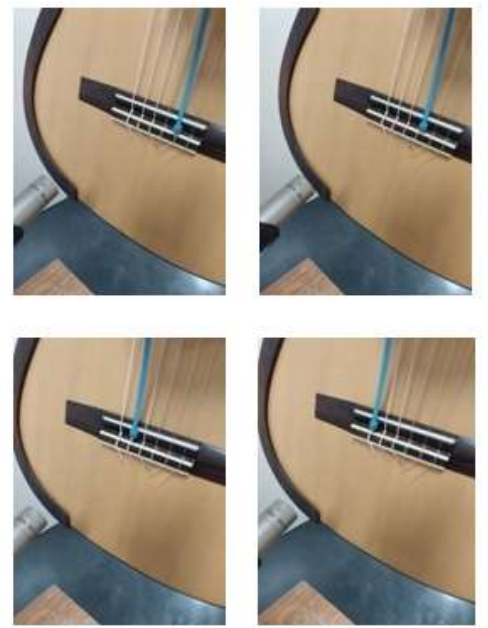

(b)

b)

Para isso o instrumento foi pendurado pela cabeça por cordas elásticas em um suporte (Figura 1 a). Nas cordas do violão foi inserida a tensão de afinação e em seguida abafadas com auxílio de espuma para que não haja interferência de vibração das mesmas, como proposto por Cossolino e Pereira (2010). Como o violão funciona com todas as suas partes acopladas, qualquer impulso faz com que todo o sistema vibre em conjunto, daí a necessidade de se colocar um elemento que absorva a vibração da corda, mas que mantenha a tensão de afinação no instrumento. E a posição deste elemento é colocado estrategicamente no braço, pois como ele é feito de material mais rígido, possivelmente irá afetar minimamente a resposta da caixa acústica.

Faz-se também uso da transformada rápida de Fourier para obtenção de gráficos do sinal no domínio da frequência observando quais entram em ressonância, até mesmo algumas que não são listadas no software do sistema sonelastic. A sequência de rotinas em matlab para obtenção dos sinais está exposta no Quadro 1. 
Quadro 1 - Sequência de Rotinas em Matlab para a Resposta em frequência pela Análise Experimental via Técnica de Excitação por Impulso.

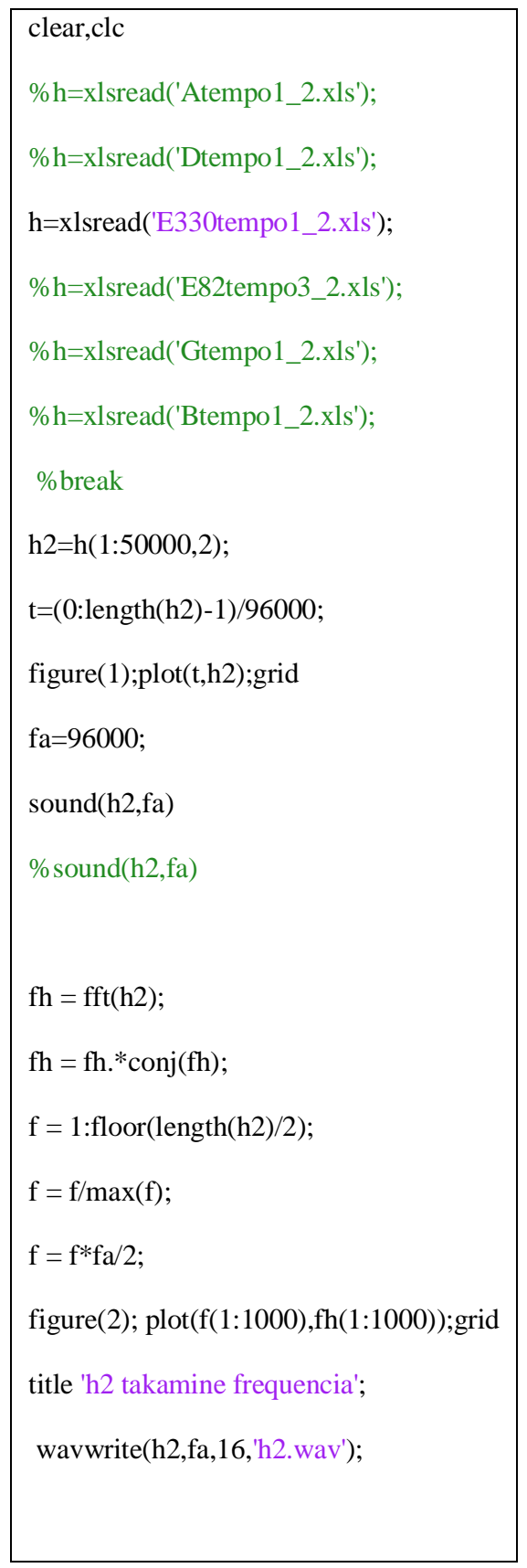

Fonte: Autores (2020).

As informações podem ser lidas na tela do software (Figura 2) ou exportadas para o Matlab em arquivos de planilha de dados em formato Excel. A rotina de comandos do quadro 1 faz inicialmente a leitura dos dados gerando um sinal sonoro de extensão wav e um gráfico no domínio do tempo seguindo para o segundo gráfico no domínio da frequência através da transformada rápida de Fourier (FFT). 
Figura 2 - Tela de aquisição de dados no software Sonelastic.

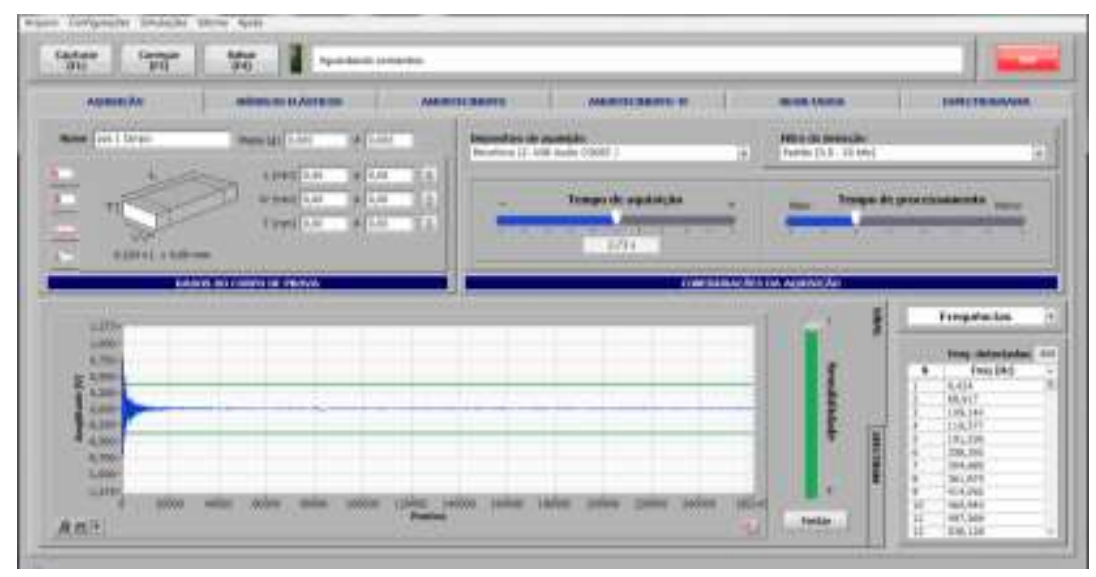

Fonte: Autores (2020).

A tela de aquisição de dados inicial do software sonelastic mostra alguns parâmetros que podem ser ajustados para uma melhor apuração dos resultados tais como tempo de aquisição, tempo de processamento, sensibilidade do microfone e o filtro de deteç̧ão. No presente trabalho são usados os ajustes como mostrado na Figura 2. Com isso, conseguiu-se uma quantidade maior de frequências de vibração para o intervalo adotado.

\subsection{Caracterização da Resposta em Frequência Através da Deconvolução}

O objetivo desse método é fazer a deconvolução dos sinais sonoros do violão pelo sinal das cordas para obter a resposta em termos de frequência do instrumento com auxílio do software Matlab. Para tal, foram gravadas respostas sonoras do violão utilizando o mesmo jogo de cordas usadas na caracterização experimental pela técnica de excitação por impulso.

Figura 3 - (a) Cordas abafadas com espuma; (b) Dispositivo para Simular braço do Violão; (c) Afinação da corda no dispositivo.

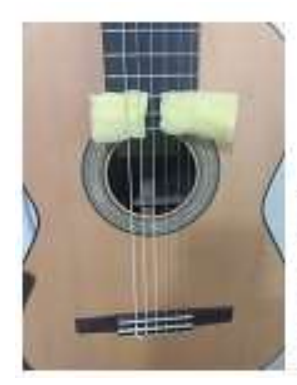

(a)

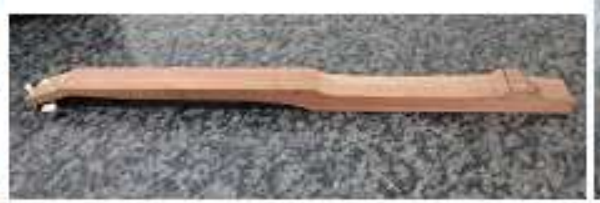

(b)

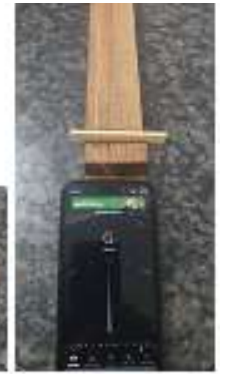

(c)

Fonte: Autores (2020).

O método consiste, inicialmente, em produzir respostas sonoras do violão a partir de dedilhamento de notas musicais com base na escala musical para instrumentos de afinação temperada. Para isso, cada corda foi tocada separadamente, abafando-se as demais com auxilio de pequenos pedaços de espuma para não haver interferência de vibração das demais (Figura 3 a).

Foi elaborado também um dispositivo com as medidas referentes ao braço do instrumento prolongando-se até o cavalete para obtenção das respostas sonoras das cordas sem a presença da caixa acústica denominado "monocórdio" (Figura 3 
b). Neste instrumento são montadas as mesmas cordas, uma de cada vez, usadas no violão com a mesma tensão de afinação usada (Figura $3 \mathrm{c}$ ).

Para captação das respostas sonoras foi usado microfone condensador Rohs $S F-910$ e, para a gravação foi usado o software Cool Edit 2000 gerando sinais com extensão wav.

A primeira etapa depois da gravação é a transferência dos sinais sonoros para serem lidos no software Matlab, gerando-se gráficos de amplitude versus tempo. Em seguida é elaborada uma série de rotinas de comandos para processar os sinais do violão e da corda para a obtenção do sinal resposta através da operação de deconvolução.

A primeira rotina elaborada é para a leitura do sinal wav da corda no violão e com a transformada rápida de Fourier (FFT) obtêm-se os sinais sonoros no domínio do tempo e frequência respectivamente (Quadro 2). Em exemplo, está demonstrado a aplicação para a corda Mí (E) ou primeira corda.

Quadro 2 - Rotinas Matlab para sinal de extensão wav e FFT do sinal sonoro da corda no violão.

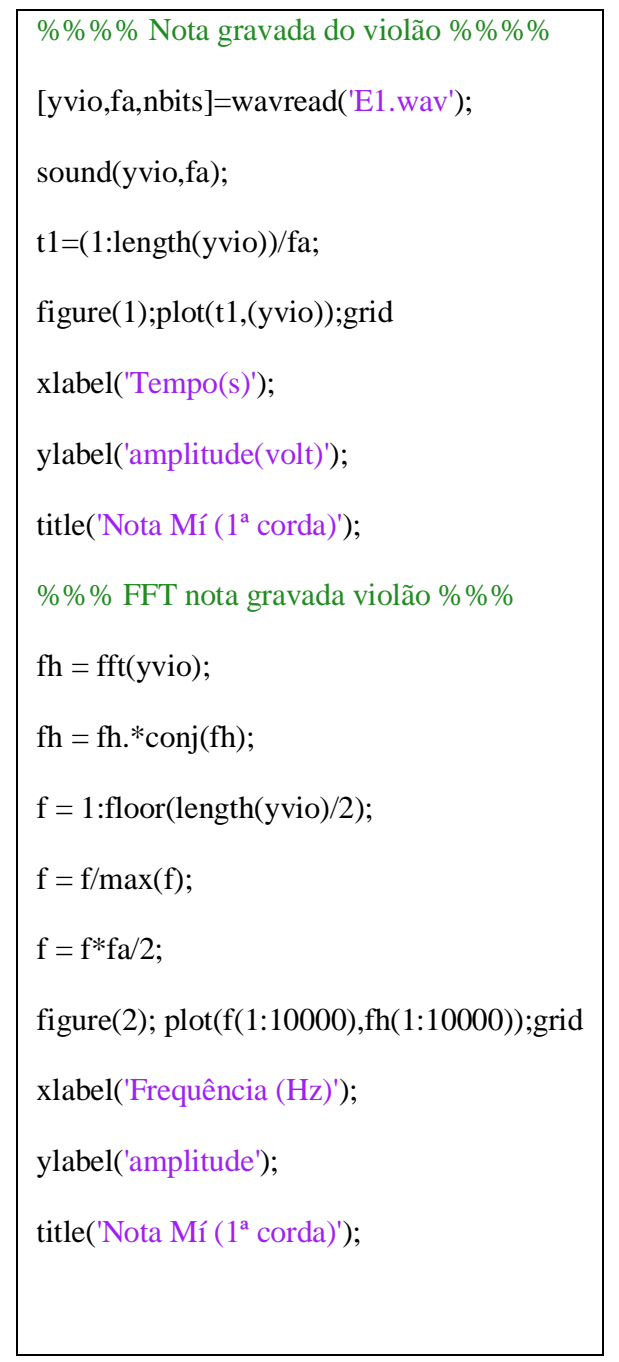

Fonte: Autores (2020).

A primeira parte da rotina de comandos do quadro 2 se refere à leitura no Matlab do sinal gravado da primeira corda (Mí) no violão usando o software Cool Edit 2000, de extensão wav obtendo a seguir a resposta no domínio do tempo e no domínio da frequência através da FFT. Com isso, obtém-se gráficos nos respectivos domínios para as posteriores análises.

Em seguida é repetido o mesmo procedimento para a mesma corda no dispositivo que simula o braço do violão sem a caixa acústica obtendo-se também os sinais sonoros nos domínios do tempo e frequência (Quadro 3 a). 
A etapa final consiste na rotina para obter o resultado da deconvolução do sinal da corda no violão pelo sinal gerado no dispositivo que simula o braço obtendo-se assim a resposta do instrumento no domínio do tempo e frequência através da FFT (Quadro 3 b).

Quadro 3 - Rotinas Matlab para sinal de extensão wav e FFT do: (a) Para a corda no dispositivo que simula o braço do violão sem a caixa acústica; (b) Para obtenção da resposta do violão nos domínios do tempo e frequência pela Deconvolução.

(a)

(b)

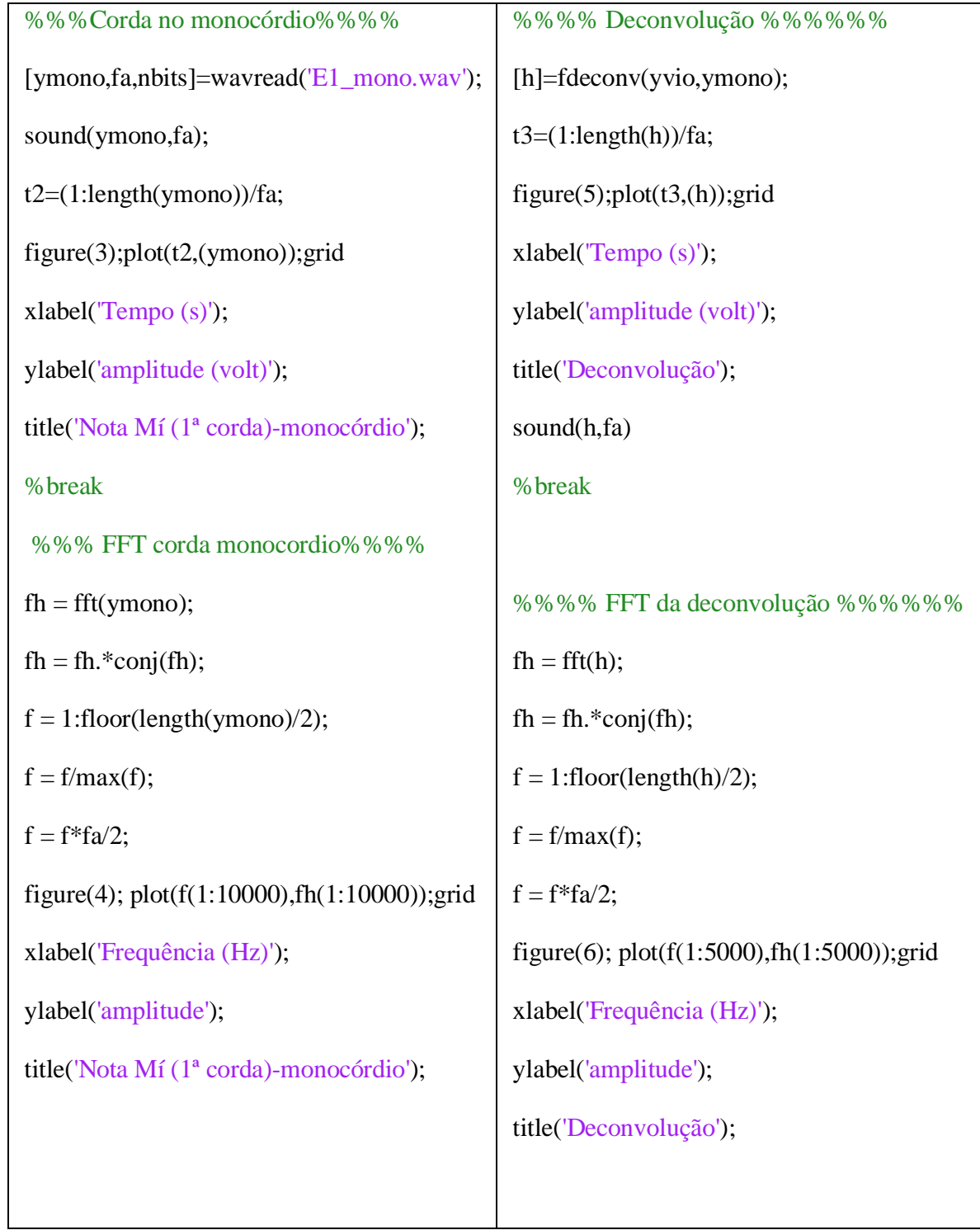

Fonte: Autores (2020).

A primeira parte da rotina de comandos do Quadro 3 (a) se refere à leitura no Matlab do sinal gravado da primeira corda (Mí) no dispositivo auxiliar (monocórdio) usando o software Cool Edit 2000, de extensão wav obtendo a seguir a resposta no domínio do tempo e no domínio da frequência através da FFT. Já no quadro 3 (b) segue uma sequência de rotinas para obtenção das respostas no domínio do tempo e frequência através de uma função característica do matlab para realização da operação de deconvolução, a fdeconv. Com isso, obtém-se também gráficos nos respectivos domínios para as posteriores análises. 


\section{Resultados e Discussão}

Na Tabela 1 são apresentados os resultados obtidos a partir dos ensaios realizados pela técnica de excitação por impulso através do software sonelastic. Foram feitos ensaios com aplicação de força impulsiva manual (batida) da primeira a sexta corda com auxilio de um instrumento feito de plástico com ponta metálica capaz de promover o impulso necessário para provocar a resposta do modelo de violão.

Tal procedimento foi feito nas posições referentes às cordas fazendo excitação no cavalete e variando o grau de sensibilidade do microfone para obtenção do máximo de frequências ressonantes. E assim foram enumeradas as vinte primeiras frequências relacionadas pelo software (Figura 4) para cada ponto de excitação e os resultados mostram que a sequência de frequências naturais listadas em ordem crescente são diferentes para cada ponto impulsionado.

Figura 4 - Tela do software sonelastic com resultados referentes a posição da primeira corda do violão (“Mí”).

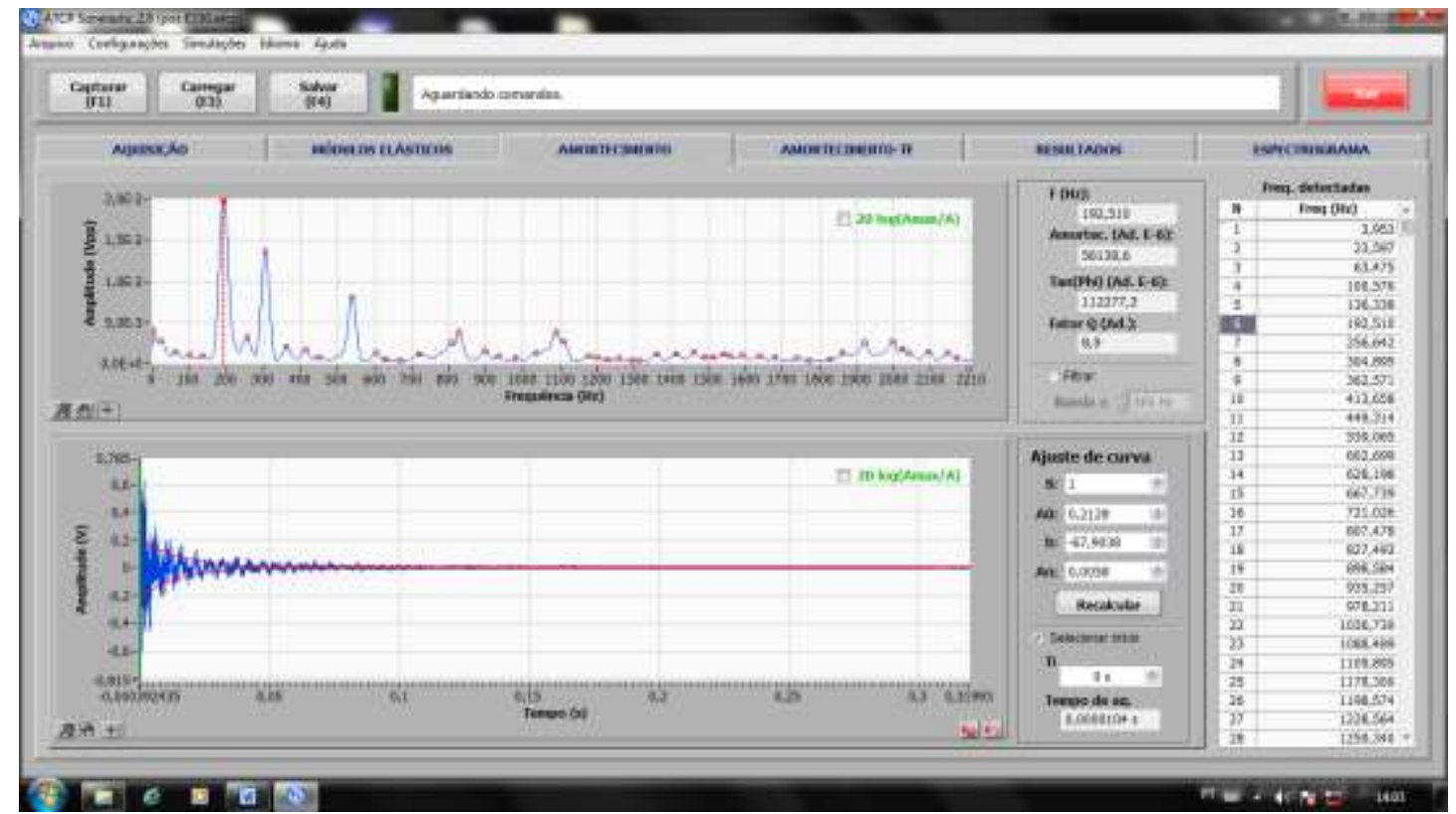

Fonte: Autores (2020).

Para cada excitação feita buscou-se variar a posição no cavalete de acordo com posição das seis cordas do violão. Com isso foram obtidas respostas no domínio do tempo e frequência através do software do sistema Sonelastic como demostrado na Figura 4, mas para uma melhor observação dos resultados, as informações foram salvas em formato de planilha de dados do Excel e transferidas para o Software Matlab para serem processados possibilitando melhores análises dos sinais gerados. E assim pode-se analisar melhor, por exemplo, as frequências de baixas amplitudes que podem não ser captadas pelo software Sonelastic, tempo de duração do sinal gerado pelo impulso.

A vibração tende a se propagar para o todo o instrumento, mas quando se muda a posição da força impulsiva inicial provavelmente o caminho de propagação possa mudar. E isso, provavelmente se deve ao fato de a madeira ser um material essencialmente anisotrópico com variações de direção do alinhamento das fibras podendo fazer com que essa vibração encontre regiões com taxas de amortecimentos diferentes e isso pode representar um fator de influência na ressonância dessas frequências.

Outro fato que se deve considerar é que a excitação para provocar a vibração foi feita manualmente, já que o pulsador automático do Sistema Sonelastic poderia causar danos a estrutura do instrumento e isto pode fazer variar os resultados em função da força aplicada. 
Tabela 1 - Respostas em frequências naturais (em Hertz) à excitação por impulso no cavalete em posições relativas às cordas do modelo numeradas em ordem crescente.

\begin{tabular}{|c|c|c|c|c|c|c|}
\hline Frequências & $\begin{array}{c}1^{a} \text { corda } \\
\text { (E) }\end{array}$ & $\begin{array}{c}2^{a} \text { corda } \\
\text { (B) }\end{array}$ & $\begin{array}{c}3^{a} \text { corda } \\
\text { (G) }\end{array}$ & $\begin{array}{c}4^{a} \text { corda } \\
\text { (D) }\end{array}$ & $\begin{array}{c}5^{a} \text { corda } \\
\text { (A) }\end{array}$ & $\begin{array}{c}6^{a} \text { corda } \\
\text { (E) }\end{array}$ \\
\hline 1 & 3,953 & 9,784 & 4,646 & 9,442 & 4,338 & 20,172 \\
\hline 2 & 23,597 & 70,338 & 69,901 & 89,060 & 56,640 & 70,941 \\
\hline 3 & 63,475 & 102,766 & 92,571 & 147,851 & 105,550 & 99,651 \\
\hline 4 & 100,576 & 137,289 & 192,634 & 191,068 & 118,220 & 132,679 \\
\hline 5 & 136,338 & 190,890 & 257,160 & 219,137 & 164,239 & 193,288 \\
\hline 6 & 192,510 & 249,427 & 302,668 & 258,686 & 197,861 & 219,093 \\
\hline 7 & 256,642 & 305,012 & 360,474 & 306,623 & 215,133 & 260,380 \\
\hline 8 & 304,895 & 350,563 & 413,173 & 332,531 & 250,561 & 305,133 \\
\hline 9 & 362,571 & 413,471 & 477,488 & 361,347 & 307,605 & 361,749 \\
\hline 10 & 413,658 & 455,628 & 539,333 & 411,469 & 363,989 & 416,135 \\
\hline 11 & 449,314 & 482,771 & 602,500 & 475,511 & 402,221 & 449,04 \\
\hline 12 & 539,065 & 540,706 & 629,093 & 540,667 & 417,720 & 471,173 \\
\hline 13 & 602,699 & 604,030 & 644,547 & 592,609 & 445,073 & 493,196 \\
\hline 14 & 628,198 & 631,524 & 664,226 & 630,063 & 492,568 & 539,018 \\
\hline 15 & 667,739 & 679,142 & 708,599 & 654,407 & 503,426 & 604,286 \\
\hline 16 & 721,026 & 724,237 & 736,791 & 680,473 & 540,275 & 626,075 \\
\hline 17 & 807,478 & 764,435 & 774,462 & 712,892 & 618,211 & 653,653 \\
\hline 18 & 827,493 & 806,217 & 825,948 & 750,803 & 651,910 & 666,648 \\
\hline 19 & 898,584 & 822,854 & 908,166 & 777,706 & 676,688 & 736,516 \\
\hline 20 & 935,257 & 846,262 & 951,433 & 800,172 & 713,284 & 779,070 \\
\hline
\end{tabular}

Fonte: Autores (2020)

Na Tabela 1 estão listadas em colunas as vinte primeiras frequências de vibração (respostas) em ordem crescente listadas pelo software sonelastic geradas a partir do impulso feito de maneira manual no cavalete nas posições relativas da primeira à sexta corda do violão.

No contexto geral, essa técnica deve fazer com que se encontrem todas as possíveis frequências ressonantes do material e a Tabela 1 nos mostra conjuntos relevantes de frequências para cada ponto excitado. Embora parte da energia possa ser perdida no caminho dessa vibração ao variar o ponto inicial, é possível encontrar varias frequências ressonantes com valores bem próximos, que possivelmente possa ser de uma mesma região do instrumento ou do mesmo modo de vibração.

Para melhor análise as respostas em frequência foram separadas em faixas com valores próximos para cada corda e em seguida foi calculada a média e feita a comparação com frequências homônimas (próximas) (Tabela 2). 
Research, Society and Development, v. 10, n. 1, e59810112179, 2021

(CC BY 4.0) | ISSN 2525-3409 | DOI: http://dx.doi.org/10.33448/rsd-v10i1.12179

Tabela 2 - Frequências homônimas experimental.

\begin{tabular}{cccccccc}
\hline Frequências & $1^{\text {a }}$ corda & $2^{\text {a }}$ corda & $3^{\text {a }}$ corda & $4^{\text {a }}$ corda & $5^{\text {a }}$ corda & $6^{\text {a }}$ corda & Média \\
\hline 1 & & 70,338 & 69,901 & & & 70,941 & $\mathbf{7 0 , 3 9}$ \\
\hline 2 & 100,576 & 102,766 & & & 105,550 & 99,651 & $\mathbf{1 0 2 , 1 3}$ \\
\hline 3 & 136,338 & 137,289 & & & & 132,679 & $\mathbf{1 3 5 , 4 3}$ \\
\hline 4 & 192,510 & 190,890 & 192,634 & 191,068 & & 193,288 & $\mathbf{1 9 2 , 0 7}$ \\
\hline 5 & 304,895 & 305,012 & 302,668 & 306,623 & 307,605 & 305,133 & $\mathbf{3 0 5 , 3 2}$ \\
\hline 6 & & 350,563 & & 332,531 & & & $\mathbf{3 4 1 , 5 4}$ \\
\hline 7 & 362,571 & & 360,474 & 361,347 & 363,989 & 361,749 & $\mathbf{3 6 2 , 0 2}$ \\
\hline 8 & 413,658 & 413,471 & 413,173 & 411,469 & 417,720 & 416,135 & $\mathbf{4 1 4 , 2 7}$ \\
\hline 9 & 449,314 & 455,628 & & & 445,073 & 449,04 & $\mathbf{4 4 9 , 7 6}$ \\
\hline 10 & 539,065 & 540,706 & 539,333 & 540,667 & 540,275 & 539,018 & $\mathbf{5 3 9 , 8 4}$ \\
\hline 11 & 602,699 & 604,030 & 602,500 & 592,609 & & 604,286 & $\mathbf{6 0 1 , 2 2}$ \\
\hline 12 & & & 644,547 & 654,407 & 651,910 & 653,653 & $\mathbf{6 5 1 , 1 2}$ \\
\hline 13 & 667,739 & 679,142 & 664,226 & 680,473 & 676,688 & 666,648 & $\mathbf{6 7 2 , 4 8}$ \\
\hline & & & & & & & \\
\hline
\end{tabular}

Fonte: Autores (2020)

A Tabela 2 agrupa várias frequiências com valores coincidentes ou próximos de cada corda extraindo-se a média. E assim, pode-se avaliar que a coincidência de valores próximos pode ser o indicativo de que independente do ponto inicial onde é inserida a força impulsiva, as demais regiões do instrumento podem ser afetadas pela irradiação dessa vibração e responder na sua frequência natural característica.

Para as avaliações a seguir é utilizado como exemplo as informações referente à resposta à excitação da primeira corda do violão (Mí) posta à vibrar com sua tensão característica de afinação ou por impulso na posição de mesma corda no cavalete. 
Figura 5 - Sintetização do sinal Impulso na posição da primeira corda do violão. (a) No domínio do tempo; (b) Frequência de maior amplitude; (c) Algumas frequências de baixas amplitudes.
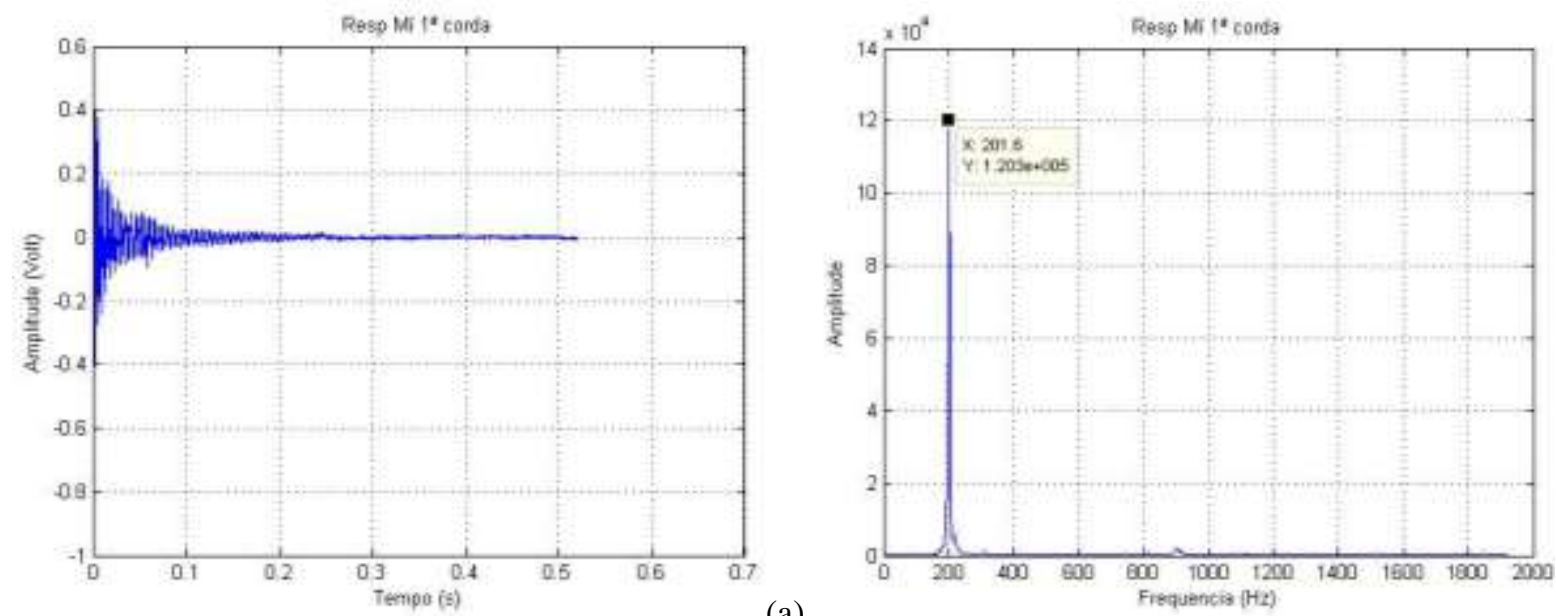

(a)

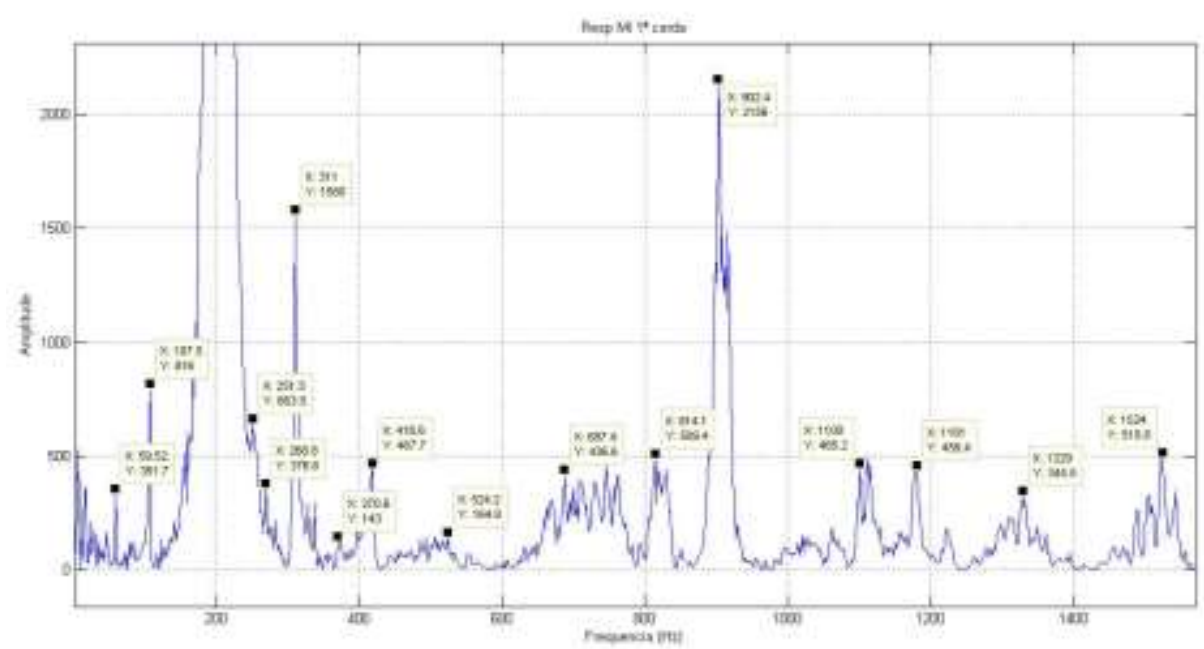

(c)

Fonte: Autores (2020)

Em exemplo, a Figura 5 (a) mostra o gráfico do sinal referente à excitação impulsiva na posição da primeira corda do violão ("Mí”) no domínio do tempo. A seguir tem-se a Figura 5 (b) evidenciando a frequência de maior amplitude (201,6 Hertz) que provavelmente possa ser da vibração inicial do impulso que irradiou gerando as demais e a Figura 5 (c) nos permite observar um conjunto maior de respostas em frequências naturais com amplitudes menores com relação à anterior com duas em maior evidência, 311 Hertz e 902,4 Hertz. Esta observação é possível devido a recursos melhores do software matlab.

As respostas em frequências lidas diretamente no software sonelastic são extremamente importantes, pois se refere aos resultados de frequências de vibração com maiores amplitudes, mas o processamento dos sinais no matlab permite que se possa relacionar um número maior de frequências, pois através de recursos do programa é possível observar até mesmo as frequências de baixa amplitude. 
Research, Society and Development, v. 10, n. 1, e59810112179, 2021

(CC BY 4.0) | ISSN 2525-3409 | DOI: http://dx.doi.org/10.33448/rsd-v10i1.12179

Tabela 3 - Respostas em frequências obtidas por excitação por impulso e deconvolução.

Respostas em Frequências (Hz)

\begin{tabular}{|c|c|c|c|c|c|c|c|c|c|}
\hline \multicolumn{5}{|c|}{ Excitação por impulso } & \multicolumn{5}{|c|}{ Deconvolução } \\
\hline \multirow{2}{*}{$\begin{array}{c}\text { Sonelastic } \\
3,9\end{array}$} & \multicolumn{4}{|c|}{ Matlab } & & & & & \\
\hline & 3,84 & 268,8 & 954,2 & 1455,0 & 8,75 & 157,1 & 535,9 & 862,5 & 1192,0 \\
\hline 23,6 & 7,68 & 282,2 & 967,7 & 1490,0 & 13,95 & 187,2 & 541,0 & 866,9 & 1216,0 \\
\hline 63,5 & 11,52 & 311,0 & 996,5 & 1503,0 & 20,55 & 190,1 & 543,9 & 958,7 & 1227,0 \\
\hline 100,5 & 17,28 & 328,3 & 1014,0 & 1509,0 & 46,98 & 191,6 & 547,6 & 960,9 & 1236,0 \\
\hline 136,3 & 23,04 & 337,9 & 1020,0 & 1523,0 & 49,92 & 197,5 & 550,5 & 986,9 & 1311,0 \\
\hline 192,5 & 34,56 & 370,6 & 1037,0 & 1542,0 & 69,73 & 199,7 & 553,5 & 1015,0 & 1315,0 \\
\hline 219,1 & 46,08 & 397,4 & 1062,0 & 1555,0 & 74,14 & 203,3 & 560,1 & 1021,0 & 1374,0 \\
\hline 256,6 & 59,52 & 418,6 & 1077,0 & 1755,0 & 80,75 & 208,5 & 576,2 & 1092,0 & 1528,0 \\
\hline 304,9 & 78,72 & 485,8 & 1100,0 & 1791,0 & 94,69 & 212,1 & 577,7 & 1106,0 & 1544,0 \\
\hline 449,3 & 84,48 & 524,2 & 1106,0 & 1809,0 & 97,63 & 218,0 & 588,7 & 1122,0 & 1637,0 \\
\hline 539,0 & 107,5 & 666,2 & 1112,0 & 1830,0 & 102,0 & 227,6 & 591,6 & 1182,0 & 1644,0 \\
\hline 602,7 & 117,1 & 687,4 & 1116,0 & 1849,0 & 103,5 & 232,0 & 600,4 & 1216,0 & 1917,0 \\
\hline 667,7 & 122,9 & 700,8 & 1129,0 & 1868,0 & 105,7 & 241,5 & 603,4 & 1220,0 & 1974,0 \\
\hline 721,0 & 136,3 & 708,5 & 1142,0 & 1874,0 & 109,4 & 247,4 & 657,7 & 1227,0 & 1995,0 \\
\hline 807,5 & 142,1 & 729,6 & 1156,0 & 1885,0 & 112,3 & 254,7 & 676,8 & 721,0 & 2013,0 \\
\hline 827,5 & 153,6 & 745,0 & 1165,0 & 1903,0 & 118,9 & 257,7 & 681,9 & 807,5 & 2013,0 \\
\hline 898,5 & 157,4 & 762,2 & 1181,0 & & 121,1 & 265,0 & 685,6 & 827,5 & 2014,0 \\
\hline 935,2 & 167,0 & 814,1 & 1221,0 & & 123,3 & 309,8 & 688,5 & 898,5 & 2018,0 \\
\hline 978,2 & 170,9 & 829,1 & 1261,0 & & 126,3 & 329,6 & 693,7 & 935,2 & 2024,0 \\
\hline 1036,7 & 182,4 & 850,6 & 1298,0 & & 129,2 & 332,5 & 733,3 & 978,2 & 2046,0 \\
\hline 1088,5 & 201,6 & 898,6 & 1309,0 & & 132,1 & 334,7 & 773,0 & 1036,7 & 2062,0 \\
\hline 1109,9 & 218,9 & 902,4 & 1327,0 & & 134,3 & 336,9 & 795,7 & 1088,5 & \\
\hline 1178,3 & 240,0 & 908,2 & 1348,0 & & 140,2 & 462,4 & 811,1 & 1109 & \\
\hline 1198,57 & 243,8 & 915,8 & 1361,0 & & 143,9 & 480,8 & 832,4 & 1178,3 & \\
\hline 1228,5 & 251,5 & 929,3 & 1394,0 & & 146,8 & 530,0 & 844,9 & 1188,0 & \\
\hline
\end{tabular}

Fonte: Autores (2020).

A Tabela 3 mostra várias frequências naturais do modelo de violão listadas em ordem crescente. Na primeira coluna estão relacionadas respostas decorrentes da excitação por impulso na posição referente à primeira corda no cavalete obtidas diretamente no Software Sonelastic e também processados no Software Matlab na segunda coluna, onde foi possível relacionar número maior de frequências. Ainda na mesma tabela, na terceira coluna, estão as respostas obtidas pelo processo de 
deconvolução do sinal sonoro do instrumento obtido ao tocar a primeira corda (Mí) pelo sinal da mesma corda tocada em instrumento auxiliar que simula o braço do violão.

Tabela 4 - Comparação de Frequências com valores próximos obtidos pela Excitação por impulso no Sonelastic e deconvolução usando Matlab.

\begin{tabular}{|c|c|c|c|}
\hline Freq. & Impulso Sonelastic & Deconvolução & Erro (\%) \\
\hline 1 & 63,5 & 69,73 & 8,93 \\
\hline 2 & 100,5 & 102,0 & 1,47 \\
\hline 3 & 136,3 & 134,3 & 1,49 \\
\hline 4 & 192,5 & 191,6 & 0,47 \\
\hline 5 & 256,6 & 257,7 & 0,43 \\
\hline 6 & 304,9 & 309,8 & 1,58 \\
\hline 7 & 449,3 & 462,4 & 2,83 \\
\hline 8 & 539,0 & 541,0 & 0,37 \\
\hline 9 & 602,7 & 603,4 & 0,12 \\
\hline 10 & 667,7 & 676,8 & 1,34 \\
\hline 11 & 721,0 & 733,3 & 1,68 \\
\hline 12 & 807,5 & 811,1 & 0,44 \\
\hline 13 & 827,5 & 832,4 & 0,59 \\
\hline 14 & 898,5 & 866,9 & 3,64 \\
\hline 15 & 935,2 & 958,7 & 2,45 \\
\hline 16 & 978,2 & 986,9 & 0,88 \\
\hline 17 & 1036,7 & 1021,0 & 1,53 \\
\hline 18 & 1088,5 & 1092,0 & 0,32 \\
\hline 19 & 1109,9 & 1106,0 & 0,35 \\
\hline 20 & 1178,3 & 1182,0 & 0,31 \\
\hline 21 & 1198,57 & 1216,0 & 1,43 \\
\hline 22 & 1228,5 & 1227,0 & 0,12 \\
\hline
\end{tabular}

Fonte: Autores (2020).

A Tabela 4 relaciona frequências com valores próximos da excitação por impulso lidas no software sonelastic e pelo método de deconvolução para comparar valores que possivelmente possa pertencer a um mesmo modo de vibração em uma região do instrumento. O modo de vibração de uma estrutura ou região da mesma se caracteriza por uma vibração em frequência natural ou característica (Wright, 1996 e Santos et al., 2013).

Vinte e duas faixas de valores são relacionadas, dos quais dezoito apresentam erro de no máximo $2 \%$, outros três em torno de 3\% evidenciando que possivelmente possam ser de mesmo modo de vibração ou de mesma região do instrumento. 
Já a Tabela 5 faz a comparação de frequências com valores próximos obtidos pela Excitação por impulso e deconvolução processando todos os sinais no Matlab. Assim se faz possível a observação de uma quantidade maior de respostas frequências naturais de vibração.

Tabela 5 - Comparação de Frequências com valores próximos obtidos pela Excitação por impulso e deconvolução usando Matlab.

\begin{tabular}{cccccccc}
\hline Freq. & Impulso & Deconvolução & Erro (\%) & Freq. & Impulso & Deconvolução & Erro (\%) \\
\hline 1 & 7,68 & 8,75 & 13,91 & 23 & 687,4 & 688,5 & 0,16 \\
\hline 2 & 11,52 & 13,95 & 21,09 & 24 & 687,4 & 688,5 & 0,16 \\
\hline 3 & 17,28 & 20,55 & 18,92 & 25 & 729,6 & 733,3 & 0,51 \\
\hline 4 & 46,08 & 46,98 & 1,95 & 26 & 762,2 & 773,0 & 1,41 \\
\hline 5 & 78,72 & 80,75 & 2,57 & 27 & 814,1 & 811,1 & 0,36 \\
\hline 6 & 107,5 & 105,7 & 1,67 & 28 & 829,1 & 832,4 & 0,40 \\
\hline 7 & 117,1 & 118,9 & 1,53 & 29 & 898,6 & 898,5 & 0,01 \\
\hline 8 & 122,9 & 123,3 & 0,32 & 30 & 954,2 & 958,7 & 0,47 \\
\hline 9 & 136,3 & 134,3 & 1,46 & 31 & 967,7 & 960,9 & 0,70 \\
\hline 10 & 142,1 & 143,9 & 1,26 & 32 & 996,5 & 986,9 & 0,96 \\
\hline 11 & 157,4 & 157,1 & 0,19 & 33 & 1014,0 & 1015,0 & 0,09 \\
\hline 12 & 182,4 & 187,2 & 2,63 & 34 & 1020,0 & 1021,0 & 0,10 \\
\hline 13 & 201,6 & 203,3 & 0,84 & 35 & 1100 & 1092 & 0,72 \\
\hline 14 & 218,9 & 218,0 & 0,41 & 36 & 1106 & 1106 & 0 \\
\hline 15 & 240,0 & 241,5 & 0,62 & 37 & 1116 & 1122 & 0,54 \\
\hline 16 & 243,8 & 247,4 & 1,47 & 38 & 1181 & 1182 & 0,08 \\
\hline 17 & 251,5 & 254,7 & 1,27 & 39 & 1221 & 1220 & 0,08 \\
\hline 18 & 268,8 & 265,0 & 1,41 & 40 & 1309 & 1311 & 0,15 \\
\hline 19 & 311,0 & 309,8 & 0,38 & 41 & 1361 & 1374 & 0,95 \\
\hline 20 & 337,9 & 336,9 & 0,29 & 42 & 1523 & 1528 & 0,33 \\
\hline 21 & 485,8 & 480,8 & 1,02 & 43 & 1542 & 1544 & 0,13 \\
\hline 22 & 524,2 & 530,0 & 1,10 & 44 & 1903 & 1917 & 0,74 \\
\hline & & & & & & & \\
\hline & & & & & & & \\
\hline
\end{tabular}

Fonte: Autores (2020)

Na Tabela 5 são encontrados quarenta e quatro faixas com valores próximos em intervalo entre zero e dois mil Hertz, dos quais trinta e nove apresentam erros menores que $2 \%$ sendo que algumas dessas faixas apresentam resultados menores ainda que $0,5 \%$, o que pode evidenciar serem de mesmo modo de vibração ou de mesma região no instrumento.

$\mathrm{O}$ grande número de frequências com valores muito próximos na comparação entre o método de excitação por impulso e deconvolução, com erros que não ultrapassam dois por cento, principalmente nas comparações acima de vinte Hertz, 
evidenciam que os dois métodos podem ser utilizados em possíveis caracterizações de materiais ou de instrumentos musicais de corda.

\section{Conclusão}

O método de análise pela excitação por impulso pode ser um bom recurso na obtenção de respostas em frequências naturais de um violão ou outro qualquer outro instrumento de corda. Se faz necessário alguns ajustes iniciais de configuração do software tais como: sensibilidade do microfone, tempo de aquisição e processamento de informações para que se consiga o maior número possível de respostas dentro de um intervalo de frequências. Para uma melhor apuração dos resultados este trabalho sugere que se transfira em planilhas as informações para um software que faça processamento de sinais, como o Matlab, por exemplo, pois assim é possível observar algumas frequências de baixas amplitudes que as vezes não são detectadas ou listadas pelo software do método de excitação por impulso, mas que podem ser relevantes na caracterização.

Para o presente trabalho foi possível a observação e análise de um número muito maior de frequências do modelo de violão estudado, pois tomando-se como referência um intervalo entre zero e mil Hertz, observa-se dezenove frequências ressonantes, enquanto que ao processar os sinais foi possível encontrar cinquenta e três.

A deconvolução dos sinais sonoros emitidos pelo intrumento ao tocar uma de suas cordas pelo sinal gravado de mesma corda separadamente, feita também por processamento de sinais, se mostra também bastante eficaz, pois foi possível a análise de oitenta frequências naturais ou ressonantes no intervalo anteriormente mencionado.

A avaliação com relação à frequências homônimas, que são de valores próximos, mostra que os dois métodos podem ser aplicados na caracterização de respostas em frequências naturais de vibração de instrumentos de corda, como o violão, por exemplo. Ressalva-se que os resultados obtidos pelo método excitação por impulso podem ser melhor avaliados usando processamento de sinais para se conseguir avaliar frequências de menores amplitudes.

No presente trabalho a maioria das faixas de frequências homônimas de vibração analisadas nos dois métodos apresentam erros abaixo de dois por cento evidenciando serem de mesmo modo de vibração ou de mesma região no instrumento.

O conjunto de frequências que aparecem nos resultados, tanto pela excitação por impulso, quanto pela deconvolução são resultantes de uma vibração provocada externamente que irradia para todo o instrumento indo de encontro aos microconstituintes do material do violão. Essencialmente feito de madeira e por sua condição anisotrópica, o violão responde a esse estímulo com diferentes frequências de vibração para cada uma de suas partes ou regiões que finalmente interage com o ar contido na caixa acústica deslocando-o e, assim produzindo o som.

A escolha de bons materiais influencia diretamente na qualidade do instrumento, pois esse conjunto de respostas em frequências naturais de vibração é o que diferencia cada tipo de violão ou outro instrumento de corda dando lhes diferentes timbres.

A utilização da técnica de deconvolução pode ser sugerida para trabalhos futuros em outros tipos de instrumentos de corda tais como: cavaquinho, viola caipira, banjo, ou até mesmo violino, desde que se consiga desenvolver um dispositivo semelhante ao utilizado no presente trabalho para obter os sinais sonoros da corda separadamente do instrumento. Outra sugestão é usar o instrumento auxiliar para obtenção do sinal da corda, a excitação por impulso para obter o sinal resposta em frequências de vibração do instrumento e através de processamento de sinais fazer a convolução dos dois sinais para se obter o sinal sonoro de mesma corda tocada no violão. 
Research, Society and Development, v. 10, n. 1, e59810112179, 2021

(CC BY 4.0) | ISSN 2525-3409 | DOI: http://dx.doi.org/10.33448/rsd-v10i1.12179

\section{Referências}

Astm International (2007). Standard Test Method for Dynamic Young's Modulus, Shear Modulus, and Poisson's Ratio by Impulse Excitation of Vibration. ASTM E 1876, 15.

Avitabile, P. (2001). Experimental modal analysis. Sound and Vibration, 35, 20-31.

Baqersad, J., Poozesh, P., Niezrecki, C., \& Avitabile, P. (2014). Comparison of modal parameters extracted using MIMO, SIMO, and impact hammer tests on a three-bladed wind Turbine, Topics in Modal Analysis II, Volume 8, Springer, 185-197.

Carcagno, S., Bucknall, R., Woodhouse, J., Fritz, C. \& Plack, C. J. (2018). Effect of back wood choice on the perceived quality of steel-string acoustic guitars. The Journal of the Acoustical Society of America 144, 3533 (2018), 10.1121/1.5084735

Cossolino L. C. \& Pereira A. H. A. (2010). Amortecimento: classificação e métodos de determinação (Informativo Técnico Científico). Universidade de São Carlos.

Elejabarrieta, M. J., Ezcurra, A. \& Santamaría, C. (2000). Evolution of the vibrational behavior of a guitar soundboard along successive construction phases by means of the modal analysis technique. J. Acoust. Soc. Am., 108(1), 369-378.

Haykin, S. S. \& Van Veen, B. (2003). Sinais e sistemas. Bookman.

Katznelson, Y. (1976). An introduction to Harmonic Analysis. (2a ed.), Dover.

Lacerda, O. (1996). Compêndio de teoria elementar da música. (9a ed.), Ricordi Brasileira S.A.

Lazzarini, V. E. P. (1998). Elementos de acústica. Music Department - National University of Ireland, Maynooth.

Ludwigsen, D. (2013). Spectral character of the resonator guitar. The Journal of the Acoustical Society of America, 134.

Mendes, H. M. D., Senra, R. E. F., Junior, J. G. M., Lima, S. S., \& Mello, G. J. (2020). Sound waves in science education: as a learning facilitator. Research, Society and Development, 9(3), e03932261. https://doi.org/10.33448/rsd-v9i3.2261

O'Haver, T. (2008). A Pragmatic Introduction to Signal Processing. Department of Chemistry and Biochemistry, University of Maryland at College Park. https://terpconnect.umd.edu/ toh/spectrum/

Otani, L.B., Segundinho, P.G.A., Morales, E. A. M. \& Pereira, A.H.A. (2017). Caracterização dos módulos elásticos de madeiras e derivados utilizando a Técnica de Excitação por Impulso (ITC-05 /ATCP). ATCP Engenharia Física.

Pereira, A. S. et al. (2018). Metodologia da pesquisa científica. Ed. UAB/NTE/UFSM.

Portela, M. S. (2014). Estudo das Propriedades Acústicas da Madeira Amazônica Marupá para Tampo de Violão. Tese (Doutorado em Engenharia Mecânica). Programa de Pós Graduação em Engenharia Mecânica da Universidade Federal de Santa Catarina.

Roebben, G., Bollen, B., Van Humbeeck, J. \& Van Der Biest, O. (1997). O. Impulse excitation apparatus to measure resonant frequencies, elastic moduli, and internal friction at room and high temperature. Review of Scientific Instruments, 68, 4511. https://doi.org/10.1063/1.1148422

Santos, E. M., Molina, C. \& Tufaile, A. P. B. (2013). Violão e guitarra como ferramentas para o ensino de física. v. 35, n. 2, 2507. Revista Brasileira de Ensino de Física.

Teixeira, P. S., Silva, A. J. \& Feiteira, J. F. S. (2014). Avaliação e comparação de características de amortecimento de sinais gerados de diferentes violões Cadernos UniFoa, 9(26), 17-30.

Teixeira, P. S., Silva, A. J. \& Feiteira, J. F. S. (2015). Análise e síntese de sinais de instrumentos dedilhados. Em estudo: O Violão. (Dissertação de Mestrado). EEIMVR, Universidade Federal Fluminense.

Yilmaz, O. (1987) Seismic data processing. SEG Investigations in Geophysics.

Wright, H. (1996). The Acoustics and Psichoacoustics of the Guitar (Ph.D.Thesis). Department of Physics and Astronomy. University of Wales.

Wuensche, C. A. (2009). Física da Música. inpe/MCT. Divisão de Astrofísica. http://www.das.inpe.br/ alex/FisicadaMusica/fismus_indice.htm. 